\title{
On the quantization of the chiral solitonic bag model
}

\author{
Namik K. Pak* \\ Department of Physics, Bilkent University, Ankara, Turkey \\ T. Yilmaz \\ Department of Physics, Middle East Technical University, Ankara, Turkey
}

(Received 21 June 1989)

\begin{abstract}
A consistent quantization scheme for the two-flavor chiral solitonic bag model with unequal quark masses is developed employing a propagator formulation.
\end{abstract}

Recently we developed a quantization scheme for the two-flavor chiral solitonic bag model, employing a propagator formalism. ${ }^{1}$ Since the original motive behind that work was to compute mass differences among the members of isospin multiplets, the quarks were taken with unequal masses, yielding a perturbative term proportional to $\Delta M_{q} I^{3}$ in the collective Hamiltonian. This work was further extended to include strong $C P$ violation into the scheme. ${ }^{2}$ In the preceding paper it is pointed out $^{3}$ that the quantization proposed in Ref. 1 is incomplete. They propose an alternative quantization scheme, employing the so-called cranking formalism.

What we would like to present in this Comment is that the flaw which marred Ref. 1 can easily be cured in the framework of the original formulation without any need to resort to alternative formulations.

The two-flavor chiral bag model is defined by

$$
\mathcal{L}=\mathcal{L}_{q} \theta(R-r)+\mathcal{L}_{m} \theta(r-R)+\mathcal{L}_{B} \delta_{B},
$$

where $^{4}$

$$
\begin{aligned}
\mathcal{L}_{q}= & \psi\left(i \gamma^{\mu} \partial_{\mu}-M\right) \psi \\
\mathcal{L}_{m}= & \frac{F_{\pi}^{2}}{16} \operatorname{tr}\left(\partial_{\mu} U^{\dagger} \partial_{\mu} U\right)+\frac{1}{32 a^{2}} \operatorname{tr}\left[U^{\dagger} \partial_{\mu} U, U^{\dagger} \partial_{v} U\right]^{2} \\
& +\frac{m_{\pi}^{2} F_{\pi}^{2}}{8\left(m_{z}+m_{d}\right)} \operatorname{tr}\left[M\left(U+U^{\dagger}-2 I\right)\right], \\
\mathcal{L}_{B}= & -\frac{1}{2}\left(\bar{\psi}_{L} U \psi_{R}+\bar{\psi}_{R} U^{\dagger} \psi_{L}\right) \\
M= & \operatorname{diag}\left(m_{u}, m_{d}\right) .
\end{aligned}
$$

The meson phase is described by the static classical field configuration $U=e^{i \tau \cdot \hat{\mathbf{x}} F(r)}$ with $F(r)$ determined by minimizing the static energy and by imposing the continuity of the axial-vector current at the bag boundary. The quark phase is described by the quantum field operator $\psi(\mathbf{x}, t)$.

The standard method ${ }^{5}$ to excite the solitonic baryon degrees of freedom, that is, to construct the low-lying quantum states above the semiclassical ground state, is to make the substitution

$$
\begin{aligned}
& U(\mathbf{x}, t)=A(t) U_{s}(\mathbf{x}) A^{\dagger}(t), \\
& \psi(\mathbf{x}, t)=A(t) \psi_{0}(\mathbf{x}, t),
\end{aligned}
$$

that is, to quantize the rotational zero modes associated with the collective variables $A(t)$. Here $U_{s}(\mathbf{x})$ and $\psi_{0}(\mathrm{x}, t)$ are the fields in the rotating (body-fixed) frame. ${ }^{3}$ Upon substituting (3) into (2), we get

$$
\begin{aligned}
L= & L_{0}+\lambda_{m} \operatorname{tr}\left(\dot{A}^{\dagger} \dot{A}\right)+\frac{i}{2} X^{a} \int d^{s} x \bar{\psi}_{0} \gamma^{0} \tau^{a} \psi_{0} \\
& -\frac{1}{2} \Delta m R^{3 a} \int d^{s} x \bar{\psi}_{0} \tau^{a} \psi_{0},
\end{aligned}
$$

where

$$
X^{a}=\operatorname{tr}\left(\tau^{a} A^{\dagger} \dot{A}\right), \quad R^{a b}=-\frac{1}{2} \operatorname{tr}\left(A^{\dagger} \tau^{a} A \tau^{b}\right) .
$$

In Eq. (4), $\lambda_{m}$ is the moment of inertia of the meson phase, associated with the collective rotations, and is given by

$$
\begin{aligned}
\lambda_{m}=\frac{2 \pi F_{\pi}^{2}}{3} \int_{R}^{\infty} & d r r^{2} \sin ^{2} F(r) \\
& \times\left\{1+\frac{4}{\left(a F_{\pi}\right)^{2}}\left[\left\{\frac{d F}{d r}\right]^{2}+\frac{\sin ^{2} F(\tau)}{r^{2}}\right]\right\} .
\end{aligned}
$$

Notice that, since the mesonic Lagrangian is at least quadratic in time derivatives, the approximation of the rotating-frame meson field with the Skyrme solution $U_{s}(\mathbf{x})$ is consistent.

In order to determine the Lagrangian (4) completely, we need to resort to the known solutions for the chiral hedgehog quark states in the equal-mass case. ${ }^{6}$ To make sensible use of these solutions in the framework of perturbation theory, we need to know the equation of motion for the rotating-frame field $\psi_{0}$. This differs, however, from the laboratory-frame equations by $A$ dependence buried in the $\psi_{0}$ 's.

Subjecting the laboratory-frame field equation $\left(i \gamma^{\mu} \partial_{\mu}\right.$ $-M) \psi=0$ to the transformation (3), we get

$$
\left(i \gamma^{\mu} \partial_{\mu}-M_{0}+i \gamma^{0} A^{\dagger} \dot{A}+\frac{1}{2} \Delta m A^{\dagger} \tau_{3} A\right) \psi_{0}(\mathbf{x}, t)=0
$$

subject to the boundary condition on the bag surface

$$
-\left.i \widehat{\mathbf{x}} \cdot \gamma \psi_{0}(\mathbf{x}, t)\right|_{\text {bag }}=\left.e^{i \gamma_{5} \hat{\mathbf{x}} \cdot \tau F(r)} \psi_{0}(\mathbf{x}, t)\right|_{\text {bag }} .
$$

Once this equation is at our disposal, its stationary-state solution $\psi_{0}(\mathbf{x}, t)=\psi_{0}(\mathbf{x}) e^{-i \omega t}$ can be related to the symmetric-case chiral hedgehog quark state solutions $\chi_{0}(\mathbf{x})$, which satisfy the equation

$$
\left(\omega \gamma_{0}+i \gamma \cdot \nabla-m_{0}\right) \chi_{0}(\mathbf{x})=0
$$

together with the boundary condition $(8) . \chi_{0}(x)$ is given as $^{5}$ 
$\chi_{0}(\mathbf{x})=\frac{N}{\sqrt{4 \pi}}\left(\begin{array}{c}i\left(\frac{E+m_{0}}{E}\right)^{1 / 2} j_{0}(k r)|0\rangle \\ -\left(\frac{E-m_{0}}{E}\right)^{1 / 2} j_{1}(k r)(\boldsymbol{\sigma} \cdot \hat{\mathbf{x}})|0\rangle\end{array}\right)$

and has some useful properties:

$$
\bar{\chi}_{0} \tau^{a} \chi_{0}=\chi_{0}^{\dagger} \tau^{a} \chi_{0}=0
$$

The relation between $\psi_{0}$ and $\chi_{0}$ is given by

$$
\begin{aligned}
\psi_{0}(\mathbf{x})= & \chi_{0}(\mathbf{x}) \\
& -\int d^{3} y S_{B}(\mathbf{x}, \mathbf{y} ; \omega)\left(i \gamma^{0} A^{\dagger} \dot{A}\right. \\
& \left.+\frac{1}{2} \Delta m_{q} A^{\dagger} \tau_{3} A\right) \psi_{0}(\mathbf{y})
\end{aligned}
$$

Here $S_{B}(x, y ; \omega)$ is the bag propagator defined by

$$
\begin{aligned}
& \left(\omega \gamma_{0}+i \gamma \cdot \nabla-m_{0}\right) S_{B}(\mathbf{x}, \mathbf{y} ; \omega)=\delta^{3}(\mathbf{x}-\mathbf{y}), \\
& {\left.\left[\exp \left(i \gamma_{5} \tau \cdot \hat{\mathbf{x}} F\right)+i \gamma \cdot \hat{\mathbf{x}}\right] S_{B}\right|_{\mathrm{bag}}=0 .}
\end{aligned}
$$

Equation (12) can be solved perturbatively to any order desired. Since $\Delta m_{q}$ is small, it is consistent to solve it to first order in $\Delta m$. Furthermore, the collective rotations are adiabatic; thus the rotational velocity $\tau^{a} A^{\dagger} \dot{A}$ is also small. Therefore, we will solve (12) to first order in the perturbation sense. To this order the rotating-frame field $\psi_{0}$ is given in terms of the symmetric hedgehog quark solutions $\chi_{0}$ as

$$
\begin{aligned}
\psi_{0}(\mathbf{x})=\chi_{0}(\mathbf{x})-\int & d^{3} y S_{B}(\mathbf{x}, \mathbf{y} ; \omega) \\
& \times\left(i \gamma^{0} A^{\dagger} \dot{A}+\frac{1}{2} \Delta m_{q} A^{\dagger} \tau_{3} A\right) \chi_{0}(\mathbf{y}) .
\end{aligned}
$$

Substituting (14) in (4), and retaining up to quadratic terms in rotational velocity $X^{a}$ (since the mesonic part is already quadratic in $X$ ) and making use of (11), we get the complete $\boldsymbol{A}$-field dependence of the Lagrangian, to first order in $\Delta m$ :

$$
L=L_{0}-\frac{1}{2} \Lambda^{a b} X^{a} X^{b}-\frac{i}{4} \Delta m R^{3 b} C^{b a} X^{a},
$$

where

$$
\begin{aligned}
& \Lambda^{a b}=\lambda_{m} \delta^{a b}+\frac{1}{2} \int d^{3} x d^{3} y\left[\bar{\chi}_{0}(\mathbf{x}) \tau^{a} \gamma_{0} S_{B}(\mathbf{x}, \mathbf{y} ; \omega) \gamma_{0} \tau^{b} \chi_{0}(\mathbf{y})+\text { H.c. }\right] \\
& C^{b a}=\int d^{3} x d^{3} y\left\{\chi_{0}^{\dagger}(\mathbf{x})\left[\tau^{a} S_{B}(\mathbf{x}, \mathbf{y} ; \omega) \tau^{b}+\tau^{b} \gamma_{0} S_{B}(\mathbf{x}, \mathbf{y} ; \omega) \gamma_{0} \tau^{a}\right] \chi_{0}(\mathbf{y})+\text { H.c. }\right\}
\end{aligned}
$$

The Hamiltonian can now be easily constructed, by taking into account the constraint $A^{\dagger} A=I$ :

$$
H=-L_{0}-\frac{1}{2} \Lambda^{a b} X^{a} X^{b} .
$$

This is consistent with the fact that for Lagrangians containing terms linear in velocity, the Hamiltonian is quadratic (to be compared with Ref. 3 ).

The spin and isospin operators can be computed in the usual manner, applying the Noether term to the transformation $\delta_{\tau} A=-i A r$ and $\delta_{l} A=i l A$ (with $r, l=\epsilon^{a} \tau^{a} / 2$ ), respectively:

$$
-S^{a}=i \Lambda^{a b} X^{b}-\frac{1}{4} R^{3 b} C^{b a} \Delta m, \quad I^{a}=R^{a b} S^{b} .
$$

By using (18), the Hamiltonian can be expressed in terms of spin and isospin operators with further neglect of the terms quadratic in $\Delta m_{q}$ :

$$
H=-L_{0}-\frac{1}{2}\left(\Lambda^{-1}\right)^{a b} S^{a} S^{b}-\frac{1}{4} \Delta m\left(R C \Lambda^{-1} R^{-1}\right)^{3 a} I^{a} .
$$

The computation of the last term, which accounts for the mass splitting among the members of isospin multiplets (in addition to the usually negligibly small electromagnetic contributions to the splitting ${ }^{7}$ ), requires the knowledge of $S_{B}$. To compute $S_{B}$ we employ, as before, the multiple reflection expansion method. ${ }^{8}$ Supported by claims in the literature, ${ }^{9}$ we will retain only the first reflection term in the expansion

$$
\begin{aligned}
S_{B}(\mathbf{x}, \mathbf{y}, \omega)= & S^{0}(\mathbf{x}, \mathbf{y}, \omega) \\
& +R^{2} \int d \Omega_{\alpha} S^{0}(\mathbf{x}, \alpha, \omega) K_{\alpha} S^{0}(\alpha, \mathbf{y}, \omega)+\cdots
\end{aligned}
$$

where

$$
K_{\alpha}=e^{i \gamma_{5} \hat{\mathbf{n}}_{\alpha} \cdot \tau F(r)}+i \hat{\mathbf{n}}_{\alpha} \cdot \gamma .
$$

Here $S^{0}$ is the usual Dirac propagator. It is expanded in partial waves employing the two-component spherical harmonics $\phi_{j l m}$ :

$$
S^{0}(\mathbf{x}, \mathbf{y}, \omega)=\sum_{j l l^{\prime} m} S_{j l l^{\prime}}^{0}\left(r, r^{\prime} ; \omega\right) \phi_{j l m}(\Omega) \phi_{j l^{\prime} m}^{\dagger}\left(\Omega^{\prime}\right),
$$

where

$$
\begin{aligned}
& S_{j l l^{\prime}}^{0}\left(r, r^{\prime} ; \omega\right)=-i k\left[\delta_{l l^{\prime}}\left(\rho_{3} \omega+m_{0}\right)\right. \\
& \left.+k\left(l^{\prime}-l\right) \rho_{2}\right] f_{l}(k r) f_{l^{\prime}}(k r), \\
& f_{l}(k r)=j_{l}(k r) \theta\left(r^{\prime}-r\right)+h_{l}^{(1)}(k r) \theta\left(r-r^{\prime}\right) .
\end{aligned}
$$

Although $S^{0}$ is diagonal in flavor space, the same is not true for the first and higher reflection term. A lengthy analysis, however, shows that both $\Lambda$ and $C$ matrices are diagonal in flavor space (although the following numerical analysis is carried out to first order only, this diagonality property holds to all orders in multiple reflection expansion). That is,

$$
\Lambda^{a b}=\delta^{a b}\left(\lambda_{m}+\lambda_{q}\right), \quad C^{b a}=\delta^{a b} C,
$$

where 


$$
\begin{aligned}
& \lambda_{q}=\frac{1}{2} \int d^{3} x d^{3} y\left[\bar{\chi}_{0}(\mathbf{x}) \gamma_{0} S_{B}(\mathbf{x}, \mathbf{y} ; \omega) \gamma_{0} \chi_{0}(\mathbf{y})+\text { H.c. }\right] \\
& C=\int d^{3} x d^{3} y\left\{\chi_{0}^{\dagger}(\mathbf{x})\left[S_{B}(\mathbf{x}, \mathbf{y} ; \omega)+\gamma_{0} S_{B}(\mathbf{x}, \mathbf{y} ; \omega) \gamma_{0}\right] \chi_{0}(\mathbf{y})+\text { H.c. }\right\}
\end{aligned}
$$

Thus the Hamiltonian can be rewritten as

$$
H=-L_{0}-\frac{S^{2}}{2\left(\lambda_{m}+\lambda_{q}\right)}-\Delta m_{q} \frac{C}{4\left(\lambda_{m}+\lambda_{q}\right)} I^{3} .
$$

After a lengthy calculation $\lambda_{q}$ and $C$ are found as

$$
\begin{aligned}
& C=-\frac{4 \widetilde{R}}{a F_{\pi} v^{4} j_{0}^{2}(v)\left\{\xi\left[v\left(1+\omega_{1}^{2}\right)-2 \omega_{1}\right]+\mu \omega_{1}\right\}} \\
& \times \int_{0}^{v} d y y^{2}\left(\int_{0}^{y} d x x^{2}\left[(\xi+\mu)^{2} j_{0}^{2}(x) j_{0}(y) n_{0}(y)-(\xi-\mu)^{2} j_{1}^{2}(x) j_{1}(y) n_{1}(y)\right]\right. \\
& +\int_{y}^{v} d x x^{2}\left[(\xi+\mu)^{2} j_{0}(x) n_{0}(x) j_{0}^{2}(y)-(\xi-\mu)^{2} j_{1}(x) n_{1}(x) j_{1}^{2}(y)\right] \\
& +v \int_{0}^{v} d x x^{2}\left[\operatorname { c o s } F \left\{(\xi+\mu) j_{0}^{2}(x) j_{0}^{2}(y)\left[(\xi+\mu)^{2} \mathcal{R}\left(h_{0}^{2}(v)\right)-v^{2} \mathcal{R}\left(h_{1}^{2}(v)\right)\right]\right.\right. \\
& \left.+(\xi-\mu) j_{1}^{2}(x) j_{1}^{2}(y)\left[(\xi-\mu)^{2} \mathcal{R}\left(h_{1}^{2}(v)\right)-v^{2} \mathcal{R}\left(h_{0}^{2}(v)\right)\right]\right\} \\
& \left.\left.-\frac{2}{3} v \sin F \mathcal{R}\left(h_{0}(v) h_{1}(v)\right)\left[(\xi+\mu)^{2} j_{0}^{2}(x) j_{0}^{2}(y)+(\xi-\mu)^{2} j_{1}^{2}(x) j_{1}^{2}(y)\right]\right]\right) \text {, } \\
& \lambda_{q}=-\frac{\tilde{R}}{a F_{\pi} v^{4} j_{0}^{2}(v)\left\{\xi\left[v\left(1+\omega_{1}^{2}\right)-2 \omega_{1}\right]+\mu \omega_{1}\right\}} \\
& \times \int_{0}^{v} d y y^{2}\left[\int _ { 0 } ^ { y } d x x ^ { 2 } \left\{(\xi+\mu)^{2} j_{0}^{2}(x) j_{0}(y) n_{0}(y)+(\xi-\mu)^{2} j_{1}^{2}(x) j_{1}(y) n_{1}(y)\right.\right. \\
& \left.+v^{2}\left[j_{1}^{2}(x) j_{0}(y) n_{0}(y)+j_{0}^{2}(x) j_{1}(y) n_{1}(y)\right]\right\} \\
& +\int_{y}^{v} d x x^{2}\left\{(\xi+\mu)^{2} j_{0}(x) n_{0}(x) j_{0}^{2}(y)+(\xi-\mu)^{2} j_{1}(x) n_{1}(x) j_{1}^{2}(y)\right. \\
& \left.+v^{2}\left[j_{1}(x) n_{1}(x) j_{0}^{2}(y)+j_{0}(x) n_{0}(x) j_{1}^{2}(y)\right]\right\} \\
& +v \int_{0}^{v} d x x^{2}\left[\operatorname { c o s } F \left\{(\xi+\mu) j_{0}^{2}(x) j_{0}^{2}(y)\left[(\xi+\mu)^{2} \mathcal{R}\left(h_{0}^{2}(v)\right)-v^{2} \mathcal{R}\left(h_{1}^{2}(v)\right)\right]\right.\right. \\
& -(\xi-\mu) j_{1}^{2}(x) j_{1}^{2}(y)\left[(\xi-\mu)^{2} \mathcal{R}\left(h_{1}^{2}(v)\right)-v^{2} \mathcal{R}\left(h_{0}^{2}(v)\right)\right] \\
& \left.-2 v^{2} j_{0}^{2}(x) j_{1}^{2}(y)\left[(\xi-\mu) \mathscr{R}\left(h_{1}^{2}(v)\right)-(\xi+\mu) \mathscr{R}\left(h_{0}^{2}(v)\right)\right]\right\} \\
& \left.\left.-\frac{2}{3} v \sin F \mathcal{R}\left(h_{0}(v) h_{1}(v)\right)\left[(\xi+\mu)^{2} j_{0}^{2}(x) j_{0}^{2}(y)-(\xi-\mu)^{2} j_{1}^{2}(x) j_{1}^{2}(y)\right]\right]\right) \text {, }
\end{aligned}
$$

where

$$
\mu=m_{0} R=\frac{m_{0}}{a F_{\pi}} \widetilde{R}, \quad v=k R, \quad \xi=E R, \quad \omega_{1}=\frac{j_{1}(v)}{j_{0}(v)} .
$$

We have evaluated the radial integrals in (27) by using the numerical solutions of the equation satisfied by the Skyrme profile $F(r)$ :

$$
\begin{aligned}
\left(\frac{1}{4} \widetilde{r}^{2}+2 \sin ^{2} F\right) F^{\prime \prime}+\frac{1}{2} \widetilde{r} F^{\prime} & +(\sin 2 F) F^{\prime 2} \\
& -\frac{1}{4} \sin 2 F-\frac{\sin ^{2} F \sin 2 F}{\widetilde{r}^{2}}=0
\end{aligned}
$$

with the boundary conditions $F(0)=\pi, F(\infty)=0$, and $\tilde{r}=a F_{\pi} r$. Taking $\Delta m_{q}=3.8 \mathrm{MeV}, \mu=0.5$, and $a=5.45$, we have plotted $\Delta m_{q} C / 4\left(\lambda_{m}+\lambda_{q}\right)$ as a function of the bag radius $R$ in Fig. 1. Notice that apart from some negligibly small fluctuations around $R \sim 0.2 \mathrm{fm}$ (which is probably due to the fact that we truncate our expansion at the first reflection order), the graph for $c / 4 \lambda_{\text {tot }}$ goes to zero smoothly for $R \rightarrow 0$, a gratifying result which lends support on the consistency of our quantization scheme.

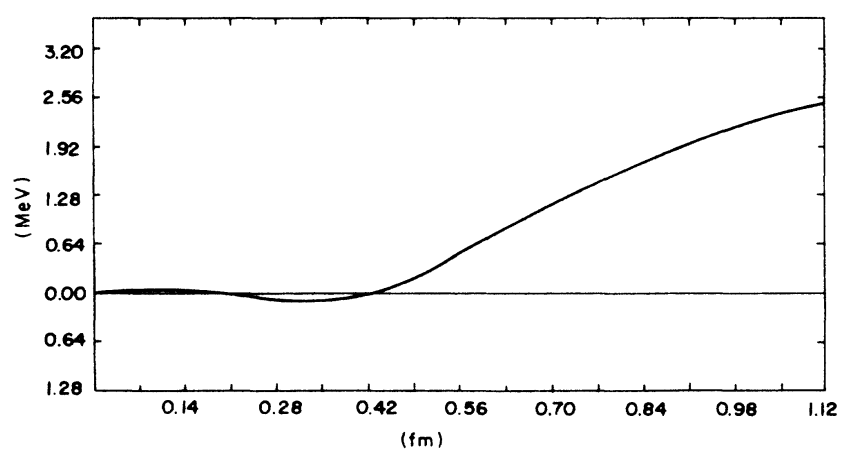

FIG. 1. The $\Delta m_{q}\left[C / 4\left(\lambda_{m}+\lambda_{q}\right)\right]$ as a function of the bag radius. 
We would like also to give the simplified expression for the spin operator for completeness (to be compared against Ref. 3):

$$
-S^{a}=i\left(\lambda_{m}+\lambda_{q}\right) X^{a}-\frac{1}{4} \Delta m C R^{3 a} .
$$

Notice that the spin is partitioned between the meson and quark sectors, as expected. Thus the inconsistency encountered in the previous attempt ${ }^{1}$ in this respect is cured as well. We differ from Ref. 3 in the quark content of the spin; their spin is insensitive to quark mass difference. Whereas in our case

$$
-S_{(q)}^{a}=i \lambda_{q} \operatorname{tr}\left(\tau^{a} A^{\dagger} \dot{A}\right)-\frac{1}{4} \Delta m C R^{3 a}
$$

That there exists a $\Delta m$-dependent term in $S_{(q)}^{a}$ is quite natural; it follows from the fact that the solutions for the well-defined spin-isospin states (baryons) are no longer solutions to the Laplace equation ${ }^{4}$ on the three-sphere. But they are solutions to the equations

$$
\begin{array}{r}
\nabla_{(4)}^{2}+i C \Delta m\left[a_{0} \frac{\partial}{\partial a_{3}}-a_{3} \frac{\partial}{\partial a_{0}}+a_{2} \frac{\partial}{\partial a_{1}}\right. \\
\left.\left.-a_{1} \frac{\partial}{\partial a_{2}}\right)\right] \Psi(a)=0
\end{array}
$$

expressed in terms of the quaternionic variables defined by $A=a_{0}+i \mathbf{a} \cdot \tau$.
*On sabattical leave from Middle East Technical University, Ankara, Turkey.

${ }^{1}$ M. Durgut, N. K. Pak, and T. Yilmaz, Phys. Rev. D 36, 3443 (1987).

${ }^{2}$ R. S. Wittman and R. M. Woloshyn, Phys. Rev. D 38, 398 (1988).

${ }^{3}$ B. Y. Park and M. Rho, preceding paper, Phys. Rev. D 41, 310 (1990).

${ }^{4}$ We use the same convention for the metric and the $\gamma$ matrices as in J. D. Bjorken and S. D. Drell, Relativistic Quantum
Fields (McGraw-Hill, New York, 1965).

${ }^{5}$ G. Adkins, C. Nappi, and E. Witten, Nucl. Phys. B228, 552 (1983).

${ }^{6}$ P. J. Mulders, Phys. Rev. D 30, 1073 (1984).

${ }^{7}$ M. Durgut and N. K. Pak, Phys. Lett. 159B, 357 (1985); 162B, 405(E) (1985).

${ }^{8}$ T. Hansson and R. L. Jaffe, Phys. Rev. D 28, 882 (1983); J. Goldstone and R. L. Jaffe, Phys. Rev. Lett. 51, 1518 (1983)

${ }^{9}$ A. Goldhaber and R. L. Jaffe, Phys. Lett. 131B, 445 (1983). 